\title{
Angiographic features of hemorrhagic moyamoya disease with high recurrence risk: a supplementary analysis of the Japan Adult Moyamoya Trial
}

\author{
Takeshi Funaki, MD, PhD, ${ }^{1}$ Jun C. Takahashi, MD, PhD, ${ }^{2}$ Kiyohiro Houkin, MD, PhD, ${ }^{3}$ \\ Satoshi Kuroda, MD, PhD, ${ }^{4}$ Shigekazu Takeuchi, MD, PhD, ${ }^{5}$ Miki Fujimura, MD, PhD, ${ }^{6}$ \\ Yasutake Tomata, $\mathrm{PhD},{ }^{7}$ and Susumu Miyamoto, MD, PhD, ${ }^{1}$ \\ on behalf of the JAM Trial Investigators
}

\begin{abstract}
${ }^{1}$ Department of Neurosurgery, Kyoto University Graduate School of Medicine, Kyoto; ${ }^{2}$ Department of Neurosurgery, National Cerebral and Cardiovascular Center, Suita; ${ }^{3}$ Department of Neurosurgery, Hokkaido University Graduate School of Medicine, Sapporo; ${ }^{2}$ Department of Neurosurgery, Graduate School of Medicine and Pharmaceutical Sciences, University of Toyama; ${ }^{5}$ Department of Neurosurgery, Nagaoka Chuo General Hospital, Nagaoka; ${ }^{6}$ Department of Neurosurgery, Tohoku University Graduate School of Medicine, Sendai; and 'Division of Epidemiology, Department of Health Informatics and Public Health, Tohoku University School of Public Health, Graduate School of Medicine, Sendai, Japan
\end{abstract}

\begin{abstract}
OBJECTIVE In this paper, the authors set out to identify the angiographic features of moyamoya disease with posterior hemorrhage, which is a strong predictor of rebleeding.

METHODS This cross-sectional study used the data set of the Japan Adult Moyamoya Trial (clinical trial registration no.: C000000166 [www.umin.ac.jp/ctr/index.htm]). The panel designed the ancillary measurement of angiography at onset, classifying the collateral vessels into 3 subtypes: lenticulostriate anastomosis, thalamic anastomosis, and choroidal anastomosis. The association between each collateral and the hemorrhage site (anterior vs posterior) was assessed in the hemorrhagic hemisphere by using multivariate adjustment for potential confounders, including age, sex, and involvement of the posterior cerebral artery (PCA). The association was confirmed through topographical analysis of bleeding points.

RESULTS Among the 80 participants, 75 hemorrhagic hemispheres of 75 patients were analyzed. Lenticulostriate anastomosis was detected in $21(28.0 \%)$ hemorrhagic hemispheres, thalamic anastomosis in $22(29.3 \%)$, and choroidal anastomosis in 35 (46.7\%). Choroidal anastomosis was a factor associated with posterior hemorrhage (OR 2.77 $[95 \% \mathrm{Cl} 1.08-7.10], \mathrm{p}=0.034)$ and remained statistically significant after the multivariate adjustment $(\mathrm{OR} 2.66[95 \% \mathrm{Cl}$ $1.00-7.07], p=0.049)$. PCA involvement was also associated with posterior hemorrhage in both univariate and multivariate analyses. Topographical analysis revealed good correspondence between bleeding points associated with positive choroidal anastomosis and the anatomical distribution of the choroidal arteries, including the thalamus and the wall of the atrium.
\end{abstract}

CONCLUSIONS Choroidal anastomosis and PCA involvement are characteristic of posterior hemorrhage in moyamoya disease. Choroidal anastomosis might be considered a potential source of posterior hemorrhage at high risk of rebleeding. https://thejns.org/doi/abs/10.3171/2016.11.JNS161650

KEY WORDS moyamoya disease; intracerebral hemorrhage; bypass surgery; cerebral angiography; vascular disorders

$\mathrm{T}$ HE Japan Adult Moyamoya (JAM) Trial was a unique randomized controlled trial demonstrating the effectiveness of direct bypass surgery for hemorrhagic moyamoya disease. ${ }^{10}$ Prespecified subgroup analysis of the trial results demonstrated that posterior hemor- rhage - a hemorrhage located in the thalamus, atrium, or other structure located in the posterior half of the brainis a significant predictor of rebleeding in moyamoya disease. ${ }^{14}$ The risk of rebleeding after posterior hemorrhage is surprisingly high: the annual rebleeding rate is $17.1 \%$

ABBREVIATIONS $\mathrm{Cl}=$ confidence interval; $\mathrm{JAM}=$ Japan Adult Moyamoya; $\mathrm{OR}=$ odds ratio; $\mathrm{PCA}=$ posterior cerebral artery.

SUBMITTED June 23, 2016. ACCEPTED November 17, 2016.

INCLUDE WHEN CITING Published online April 14, 2017; DOI: 10.3171/2016.11.JNS161650. 
after nonsurgical treatment, with the 5-year risk exceeding $50 \% .{ }^{14}$ It remains unclear, however, why posterior hemorrhage is associated with such a high rebleeding risk.

An assumed cause of hemorrhage is the rupture of the fragile collateral vessels, typical of moyamoya disease, derived from the lenticulostriate artery, thalamic artery, or choroidal artery. ${ }^{3,5,11}$ Given that the higher rebleeding risk associated with posterior hemorrhage is attributable to the diversity of the collaterals, certain collaterals at high risk of rupture are most likely to be distributed posteriorly. One cross-sectional study indicated that the collaterals from the thalamic perforators or choroidal arteries are likely to be associated with such high risk. ${ }^{11}$ The topographical relationship between these collaterals and the hemorrhage site, however, has rarely been investigated. Furthermore, apart from several pioneering works, few studies have addressed the systematic angiographic classification of fragile collaterals in moyamoya disease. ${ }^{11}$

The hypothesis of the present analysis is that collaterals derived from the thalamic or choroidal artery are responsible for posterior hemorrhage. To test the hypothesis, the JAM Trial group developed an original angiographic classification of collaterals and conducted analyses to determine which type of collateral vessel was associated with posterior hemorrhage. Furthermore, we performed qualitative, topographical analyses to confirm whether the bleeding points of posterior hemorrhage actually corresponded to the areas where the responsible collaterals were distributed.

\section{Methods}

The study was approved by the ethics committees of all participating centers and was registered with the University Hospital Medical Information Network Clinical Trials Registry (clinical trial registration no.: C000000166 [www.umin.ac.jp/ctr/index.htm], 2005).

\section{Participants and Setting}

This ancillary cross-sectional study used the data set available at the time of enrollment in the JAM Trial. Detailed information on participant and setting of the JAM Trial has been presented elsewhere. ${ }^{10}$ Participants were recruited from 22 centers: 14 academic, 5 national or public, and 3 private. Patients with moyamoya disease were eligible for the study if they had experienced intracranial hemorrhage within 12 months before randomization, were 16-65 years old, were independent in daily life (modified Rankin Scale Score 0-2), had completed acute-phase treatment at least 1 month before randomization, and had been free from ischemic/hemorrhagic attack for at least 1 month. Patients with ruptured aneurysms on the circle of Willis were excluded. Moyamoya disease was diagnosed according to the guidelines proposed in $1997 .^{2}$

\section{Classification and Measurement of Collaterals}

The primary factor of interest in the present analysis was the presence of abnormal collateral vessels in the basal and periventricular regions. These abnormal vessels were measured using angiography results obtained at the time of hemorrhage onset. An expert panel, assembled for the present analysis, developed an angiographic classification system and grading for basal and periventricular collaterals (Fig. 1). This angiographic classification was based on the system proposed by Morioka et al. in $2003^{11}$ but was modified to reflect a recent discovery regarding the periventricular collateral system observed on MRI., ${ }^{3,5}$ Collateral vessels were classified into 3 types: lenticulostriate anastomosis, thalamic anastomosis, and choroidal anastomosis. The panel, not apprised of other clinical information, recorded the presence or absence of each type of collaterals in each hemisphere according to the criteria outlined below.

\section{Lenticulostriate Anastomosis}

Lenticulostriate anastomosis resembles so-called moyamoya vessels but is more specifically defined (Figs. 1A and 2A). This type is anatomically defined as an anastomosis between the lenticulostriate artery and the medial end of the medullary artery, serving as a supply route to the cortex. A positive angiographic indicator of lenticulostriate anastomosis is extreme dilation and extension of the lenticulostriate arteries with at least 1 artery extending beyond the level of the pericallosal artery in the lateral view. In such a situation, the lenticulostriate arteries are considered to connect to the medullary arteries at the lateral corner of the lateral ventricle because the position of the pericallosal artery approximates the upper margin of the lateral ventricle. In the anteroposterior view, this anastomosis is typically observed as long, laterally curved arteries with a sharp inflection at their peripheral portion (Fig. 2A upper).

\section{Thalamic Anastomosis}

Thalamic anastomosis is defined as an anastomosis between the thalamic perforator and the medial end of the medullary artery or the insular artery, serving as a supply route to the cortex (Figs. 1B, 2B, and 2C). The thalamotuberal artery, arising from the posterior communicating artery, and thalamoperforating artery, arising from the posterior cerebral artery (PCA), are the origins of such an anastomosis. A positive angiographic indicator of thalamic anastomosis is extreme dilation and extension of the thalamic perforators with at least 1 perforator extending beyond the position of the medial posterior choroidal artery in the lateral view. In such a situation, the thalamic perforators are reasonably considered to extend beyond the thalamus to connect to the medullary arteries because the position of the medial posterior choroidal artery approximates the upper margin of the thalamus.

\section{Choroidal Anastomosis}

Choroidal anastomosis is typically defined as an anastomosis between the choroidal artery and the medial end of the medullary artery (Figs. 1C and 2D). Both the anterior and lateral posterior choroidal arteries can serve as the origin of such an anastomosis. A positive angiographic (lateral view) indicator of choroidal anastomosis is extreme dilation and extension of the choroidal artery with sudden deviation from the shape of the lateral ventricle at its peripheral portion to connect to the medullary artery. In the anteroposterior view, this collateral is similar 

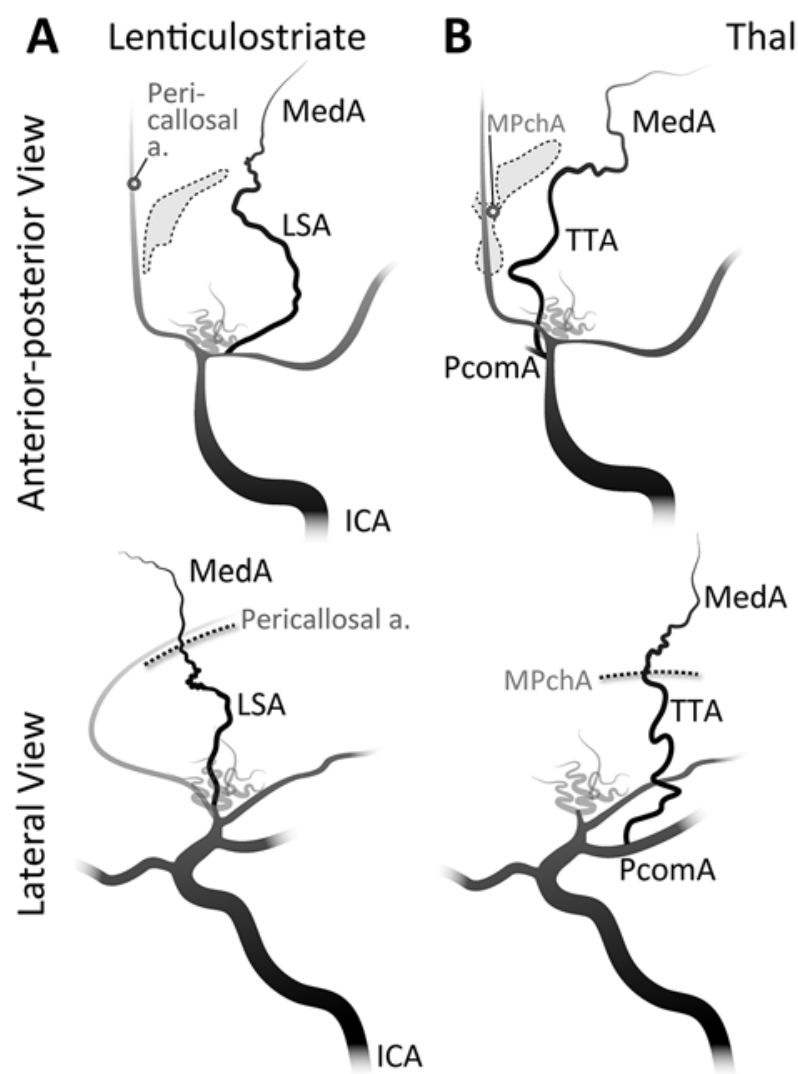

Thalamic

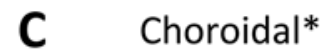

FIG. 1. Schematic illustration showing angiographic definition of lenticulostriate (A), thalamic (B; dotted lines represent the medial posterior choroidal artery), and choroidal (C) anastomoses. The gray area indicates the ventricle. *Includes collaterals from the posterior choroidal artery. The panels to the left of the dashed vertical line are representative of a carotid artery angiogram, and those to the right of this line are representative of a vertebral artery angiogram. $a .=$ artery; $C h A=$ choroidal artery; ICA = internal carotid artery; LSA = lenticulostriate artery; MedA = medullary artery; MPchA = medial posterior choroidal artery; PCA = posterior cerebral artery; PcomA = posterior communicating artery; TPA = thalamoperforating artery; TTA = thalamotuberal artery; VA = vertebral artery.

to lenticulostriate anastomosis in that it has a typical sharp inflection laterally (Fig. 2D upper). Another positive indicator is the extreme extension of the anterior choroidal or lateral posterior choroidal artery beyond the atrium of the lateral ventricle to reach the body of the lateral ventricle. Regarding the medial posterior choroidal artery, anastomosis is considered positive when the artery connects to the pericallosal artery by penetrating the corpus callosum. ${ }^{1}$

\section{Other Variables}

The location of hemorrhage, which had been classified as either anterior or posterior according to CT findings before randomization, was the primary dependent variable in the present analysis. The definition of anterior and posterior hemorrhages has been described elsewhere. ${ }^{14} \mathrm{In}$ brief, an anterior hemorrhage is defined as being located in the putamen, caudate head, frontal lobe, anterior half of the temporal lobe, subependymal area of the anterior part of the lateral ventricle, and anterior half of the corpus callosum. A posterior hemorrhage is defined as being located in the thalamus, posterior half of the temporal lobe, parietal lobe, occipital lobe, subependymal area of the posterior part of the lateral ventricle including the atrium, and posterior half of the corpus callosum.
An expert panel additionally categorized the location of the bleeding point of each hemorrhage as either right sided or left sided according to the CT findings at onset and MRI scans if available. This categorization was done separately by a different panel to minimize information bias. Each bleeding point was plotted on a schematic axial-slice brain template for analysis of the topographical relationship between the hemorrhage site and collaterals.

The following baseline data were collected: age, sex, presence of risk factors (hypertension, diabetes, and hyperlipidemia), and history of ischemic event or hemorrhagic stroke. Disease severity was defined according to the Suzuki stage, ${ }^{13}$ and the involvement of the PCA, a candidate for a potential confounder, were also measured with angiography at onset. PCA involvement was defined as the presence of occlusion or stenosis greater than $50 \%$ in the $\mathrm{P}_{1}-\mathrm{P}_{3}$ segments of the PCA with decreased delineation of the cortical arteries. ${ }^{4}$ Suzuki stage and PCA involvement were recorded for each hemisphere.

\section{Statistical Analysis}

Information on the hemorrhagic hemisphere (the hemisphere in which the hemorrhage had supposedly occurred in each patient) was extracted for the statistical analysis 

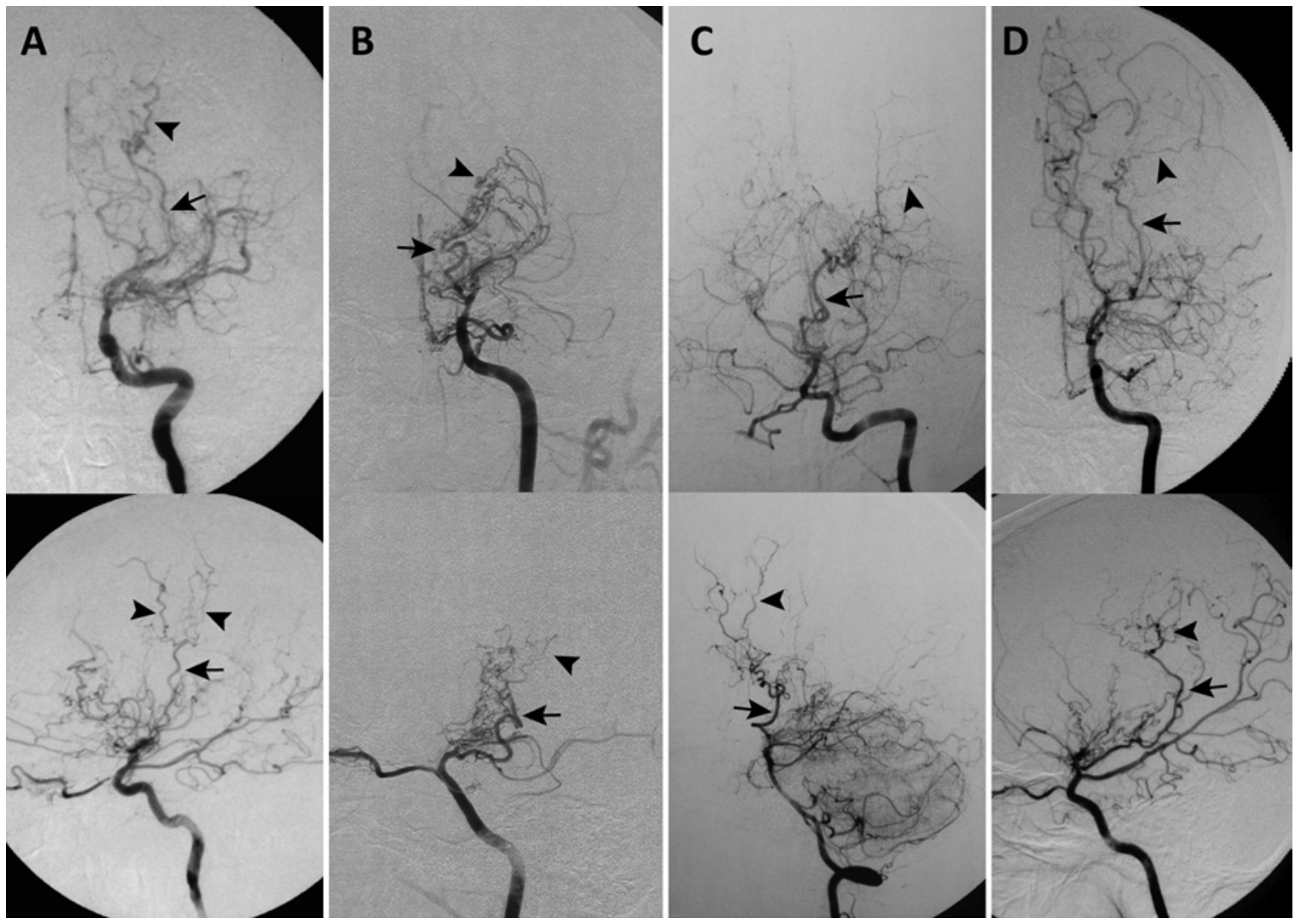

FIG. 2. Typical angiographic findings of lenticulostriate (A), thalamic (B and C), and choroidal (D) anastomoses. Anteroposterior and lateral views are shown in the upper row and lower row, respectively. The origins of thalamic anastomoses include the posterior communicating (B) and posterior cerebral (C) arteries. The perforating or choroidal arteries are indicated by arrows; the medullary arteries are indicated by arrowheads.

(Fig. 3). Patients in whom the hemorrhagic hemisphere could not be determined were excluded from the analysis. The association between the type of collateral and the location of hemorrhage was estimated as an odds ratio (OR) for posterior hemorrhage relative to anterior hemorrhage. As for comparison of the baseline characteristics, the ttest, chi-square test, and Mann-Whitney U-test were used as appropriate. Multiple logistic regression models were used for multivariate adjustment of confounders. Two-sided values of $\mathrm{p}<0.05$ or $95 \%$ confidence intervals (CIs) not

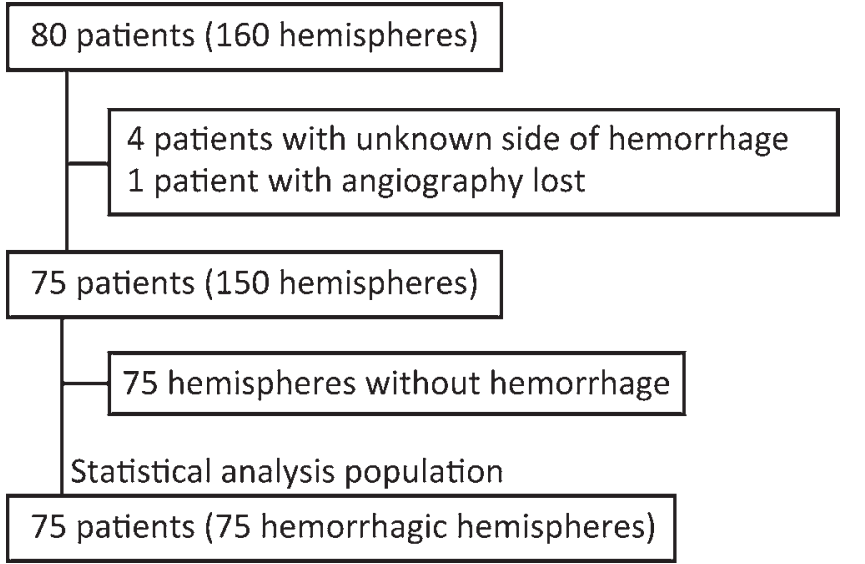

FIG. 3. Flow diagram for inclusion. including 1 were considered significant. All analyses were performed with IBM SPSS Statistics Desktop, (version 22, IBM Software Group).

\section{Results}

Among the total of 80 patients enrolled in the JAM Trial, 4 were excluded from the present analysis because the hemisphere in which their hemorrhage occurred could not be determined; specifically, in 3 of these patients the hemorrhage occurred on the midline and in the remaining patient the diffusely distributed intraventricular hemorrhage occurred in the bilateral ventricles. Another patient was excluded because the original angiography study obtained in that patient had been lost. Accordingly, 75 hemorrhagic hemispheres in 75 patients were included in the actual analysis (Fig. 3). These 75 hemispheres comprised 38 right and 37 left hemispheres and accounted for 42 anterior and 33 posterior hemorrhages.

Twenty-eight (37.3\%) hemispheres exhibited only 1 type of anastomosis, 19 (25.3\%) exhibited 2 types, and $4(5.3 \%)$ exhibited all 3 types. No anastomoses were detected in 24 hemispheres (32.0\%). Lenticulostriate anastomosis was detected in $21(28.0 \%)$ hemispheres, thalamic anastomosis in 22 (29.3\%), and choroidal anastomosis in 35 (46.7\%). PCA involvement was observed in 24 (32\%) hemorrhagic hemispheres. 
TABLE 1. Baseline variables in 75 patients with 75 hemorrhages

\begin{tabular}{lccc}
\hline \multicolumn{1}{c}{ Variable } & $\begin{array}{c}\text { Anterior } \\
\text { Hemorrhage }\end{array}$ & $\begin{array}{c}\text { Posterior } \\
\text { Hemorrhage }\end{array}$ & $\begin{array}{c}p \\
\text { Value }\end{array}$ \\
\hline No. of hemispheres & 42 & 33 & \\
\hline Mean age \pm SD, yrs & $41.2 \pm 11.3$ & $42.7 \pm 11.7$ & 0.587 \\
\hline Female sex & $15(35.7)$ & $9(27.3)$ & 0.437 \\
\hline Hypertension & $8(19.0)$ & $7(21.2)$ & 0.816 \\
\hline Diabetes mellitus & $2(4.8)$ & $1(3.0)$ & 0.704 \\
\hline Hyperlipidemia & $2(4.8)$ & $2(6.1)$ & 0.803 \\
\hline History of ischemic event & $11(26.2)$ & $10(30.3)$ & 0.694 \\
\hline History of hemorrhagic stroke & $2(4.8)$ & $6(18.2)$ & 0.062 \\
\hline Suzuki stage & \multicolumn{3}{c}{0} \\
\hline 1 & 0 & 0 & \\
\hline 2 & $2(4.8)$ & 0 & \\
\hline 3 & $13(31.0)$ & $9(27.3)$ & \\
\hline 4 & $21(50.0)$ & $14(42.4)$ & \\
\hline 5 & $6(14.3)$ & $10(30.3)$ & \\
\hline 6 & 0 & 0 & \\
\hline Median score (IQR) & $4(3-4)$ & $4(3-5)$ & 0.134 \\
\hline
\end{tabular}

Values are presented as the number of patients (\%) unless stated otherwise.

\section{Association Between Collaterals and Posterior Hemorrhage}

Table 1 summarizes the baseline characteristics of 75 patients with an anterior or posterior hemorrhage. No significant differences in baseline characteristics were observed between the 2 groups.

In the univariate analysis, choroidal anastomosis and PCA involvement were significantly associated with posterior hemorrhage (Fig. 4). The ORs of choroidal anastomosis and PCA involvement for posterior hemorrhage were 2.77 (95\% CI 1.08-7.10) and 3.06 (95\% CI 1.128.36), respectively. Although the OR of thalamic anastomosis also exceeded 1, the association between thalamic anastomosis and posterior hemorrhage was not significant (OR 1.41 [95\% CI 0.52-3.82]). In contrast, the OR of lenticulostriate anastomosis was less than 1 (OR $0.94[95 \%$ CI 0.34-2.59]).
TABLE 2. Multivariate-adjusted ORs for posterior hemorrhage

\begin{tabular}{|c|c|c|c|c|c|c|}
\hline \multirow[b]{2}{*}{$\begin{array}{c}\text { Type of } \\
\text { Anastomosis }\end{array}$} & \multicolumn{3}{|c|}{ Crude } & \multicolumn{3}{|c|}{ Multivariate Adjustment* } \\
\hline & OR & $\begin{array}{c}95 \% \\
\mathrm{Cl}\end{array}$ & $\begin{array}{c}p \\
\text { Value }\end{array}$ & OR & $\begin{array}{c}95 \% \\
\mathrm{Cl}\end{array}$ & $\begin{array}{c}p \\
\text { Value }\end{array}$ \\
\hline $\begin{array}{l}\text { Lenticulostri- } \\
\text { ate }\end{array}$ & 0.94 & $(0.34-2.59)$ & 0.901 & 0.83 & $(0.26-2.59)$ & 0.743 \\
\hline Thalamic & 1.41 & $(0.52-3.82)$ & 0.501 & 1.03 & $(0.34-3.08)$ & 0.963 \\
\hline Choroidal & 2.77 & $(1.08-7.10)$ & 0.034 & 2.66 & $(1.00-7.07)$ & 0.049 \\
\hline
\end{tabular}

The estimate of each collateral was adjusted by 3 possible confounders - sex, age, and PCA involvement-with separate multiple logistic regression models (Table 2). Choroidal anastomosis remained statistically significant after the adjustment (OR 2.66 [95\% CI 1.00-7.07], $\mathrm{p}=$ 0.049). PCA involvement also remained statistically significant in the model incorporating choroidal anastomosis (OR 2.92 [95\% CI 1.01-8.46], $\mathrm{p}=0.049$; not shown).

\section{Topographical Analysis}

Figure 5A presents a plot of all bleeding points of posterior hemorrhage. Bleeding points associated with positive thalamic and/or choroidal anastomosis are separately shown in Figs. 5B-D. For ease of recognition, all bleeding points are depicted in the left hemisphere.

Generally, among all posterior hemorrhages, the bleeding points associated with positive choroidal anastomosis were most likely to be distributed posteriorly (Fig. 5B). Furthermore, the distribution corresponded closely to the anatomical distribution of the anterior and lateral posterior choroidal arteries: the posterior limb of the internal capsule, posterolateral part of the thalamus (pulvinar), and the wall of the atrium of the lateral ventricle. A bleeding point was located at the corpus callosum.

Of 4 bleeding points associated with positive thalamic anastomosis, 2 were located at the wall of the body of the lateral ventricle and 2 were at the wall of the atrium (Fig. 5C). Of 7 bleeding points associated with both thalamic and choroidal anastomoses, 4 were located at the thala-

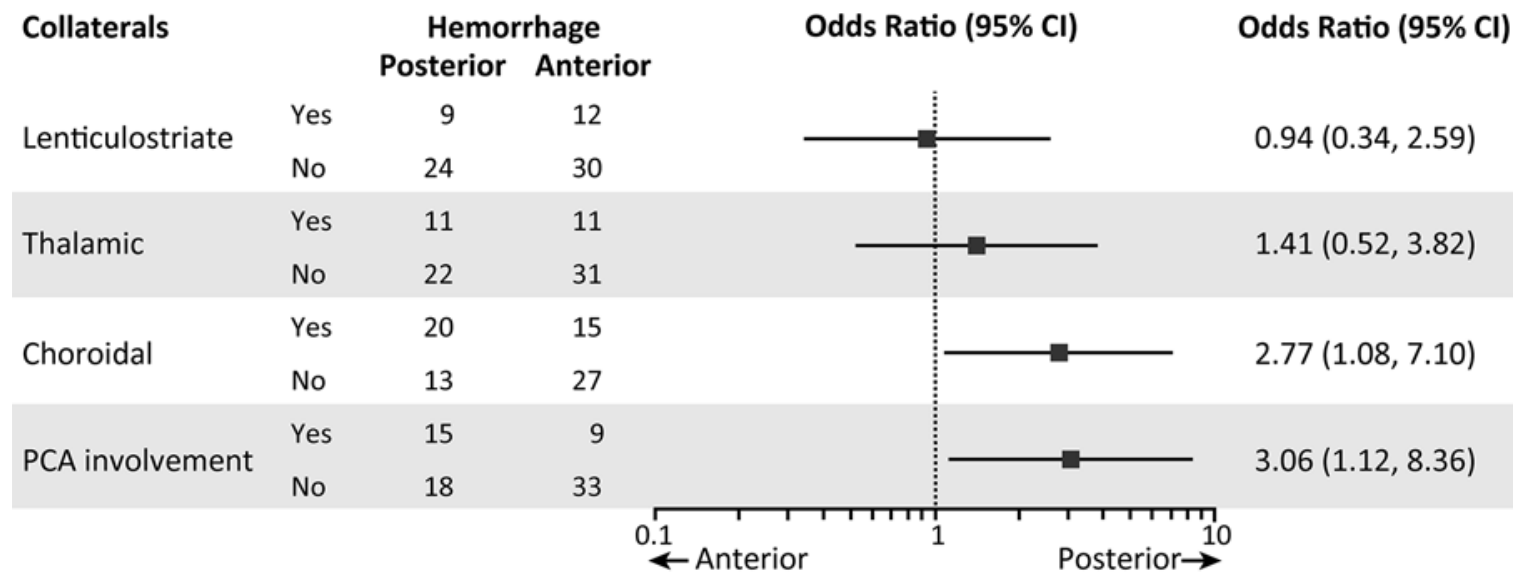

FIG. 4. Graphic display of univariate ORs for posterior hemorrhage. 
A

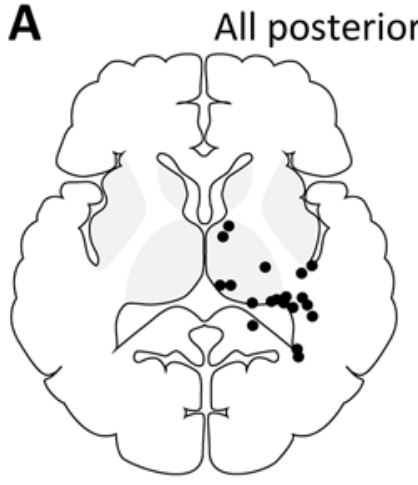

C

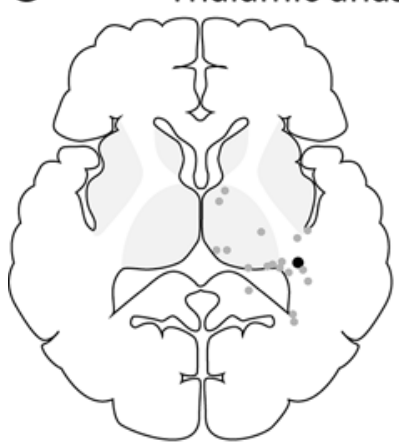

hemorrhages

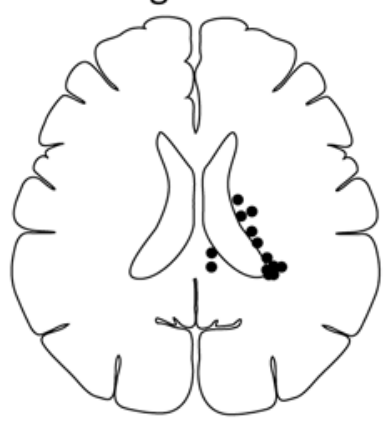

mosis positive

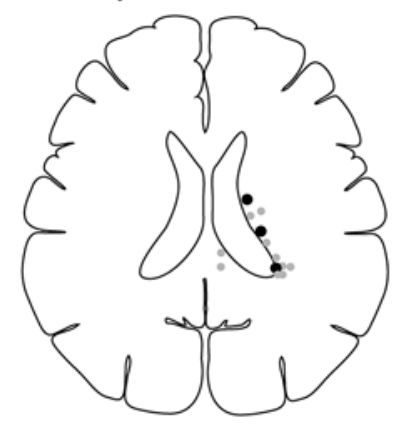

B
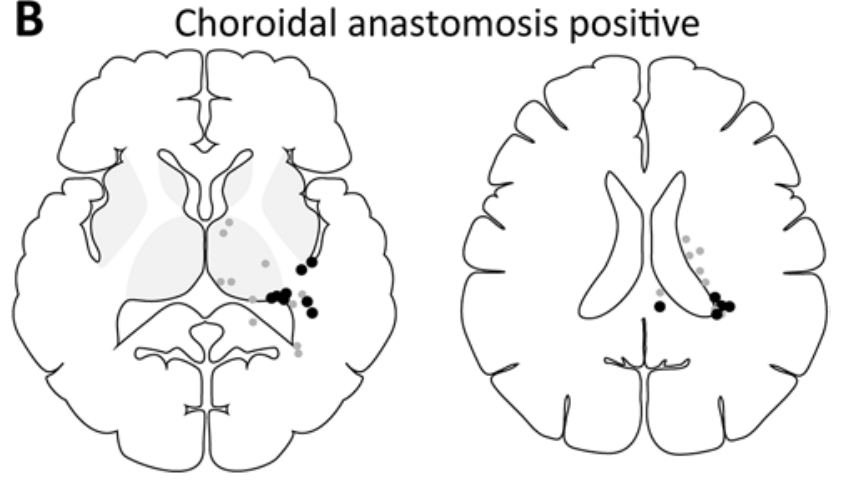

D Choroidal \& thalamic anastomosis positive
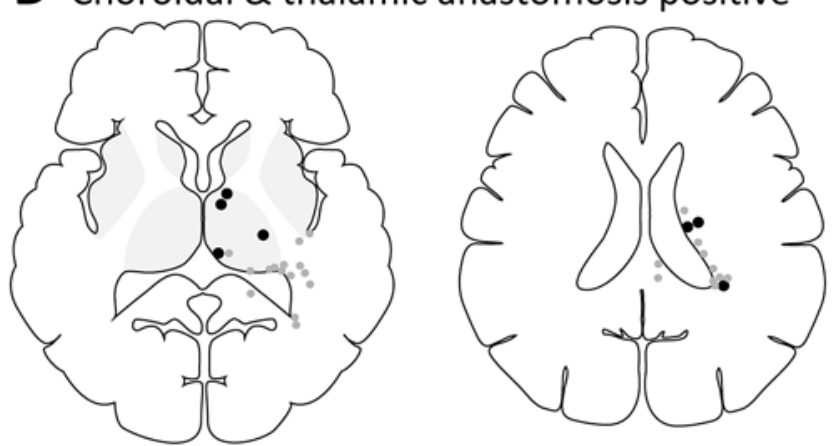

FIG. 5. Topographical analysis showing the relationship between bleeding points (gray dots) and collaterals. Black dots represent each collateral detected. For ease of recognition, all bleeding points are presented as on the left hemisphere. Gray shading indicates the basal ganglia and thalamus.

mus, 2 were at the wall of the body of the lateral ventricle, and 1 was at the wall of the atrium, corresponding to anatomical distribution of both thalamic perforators and choroidal arteries (Fig. 5D).

\section{Discussion}

Our results reveal a significant association between choroidal anastomosis and posterior hemorrhage and topographical correspondence between bleeding point and anatomical distribution of the collateral vessel, suggesting that choroidal anastomosis is primarily responsible for posterior hemorrhage. Our results also suggest that PCA involvement is another independent factor associated with posterior hemorrhage.

Our results are in line with those of several studies, implying the importance of the choroidal artery in hemorrhagic moyamoya disease. Irikura et al. reported that dilated choroidal arteries were frequently seen in hemorrhagic moyamoya disease. ${ }^{6}$ Morioka et al. demonstrated that dilation and branch extension of the anterior choroidal arteries were significantly associated with hemorrhagic presentation. ${ }^{11}$ Choroidal anastomosis in our definitionanastomosis between the choroidal and medullary arteries-is almost identical to these angiographic findings, although its anatomical features are more specifically defined. Recent studies incorporating high-definition MR angiography have demonstrated the same characteristics of the collateral vessel. 3,5

Our results suggest that, among the collaterals, choroidal anastomosis is in the most posterior location, as illustrated in Fig. 6. This result is supported by vascular anatomy. The anterior choroidal artery supplies the posterior limb of the internal capsule, part of the temporal lobe, the lateral geniculate body, and so on..$^{15}$ The lateral posterior choroidal artery supplies the most lateral region of the posterior part of the thalamus (pulvinar) and dorsolateral portion of the thalamus. ${ }^{16}$ These choroidal arteries also supply the wall of the atrium of the lateral ventricle via the subependymal branches. ${ }^{8,12}$ All of these distributions of the choroidal arteries are included in the posterior half of the brain. Although our topographical analysis detected a hemorrhage that did not correspond to the anatomical distribution of the choroidal artery (the corpus callosum), this hemorrhage could be attributable to the anastomosis between the medial posterior choroidal artery and pericallosal artery via the corpus callosum, ${ }^{1}$ a rare type of choroidal anastomosis.

Although thalamic anastomosis tends to be associated with posterior hemorrhage, the association is not statistically significant, contradicting our study hypothesis. As shown in the topographical analyses (Fig. 5), thalamic anastomosis seems to cause not only thalamic hemorrhage but also subependymal hemorrhage at the body of the lateral ventricle. According to recent studies using MRI, 3,5 thalamic perforators might extend upward and anastomose to the medullary artery at the periventricular area of the body of the lateral ventricle. The anastomosis might cluster at the anteroposterior border of the lateral ventricle, resulting in relatively even distribution of intraventricular hemorrhage. 


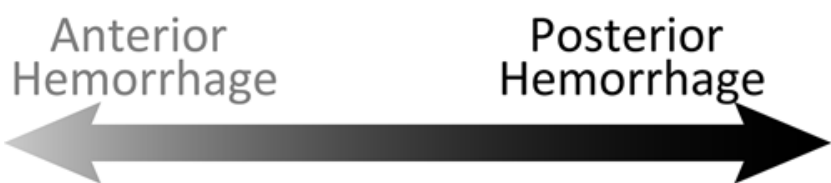

\section{Lenticulostriate Thalamic Choroidal}

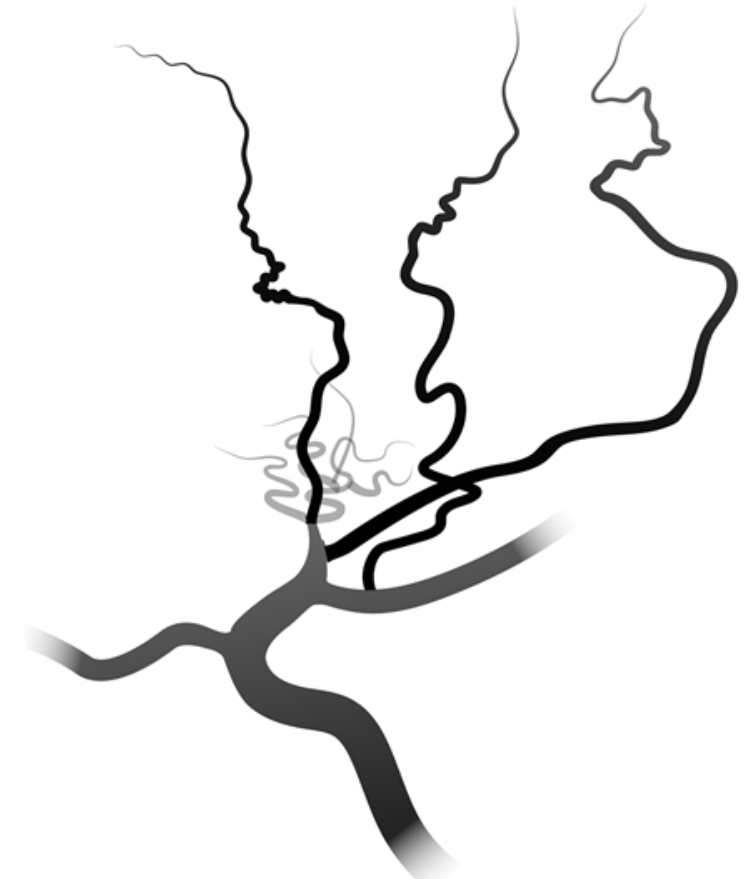

FIG. 6. Schematic illustration showing the conceptual relationship between collaterals and hemorrhage locations.

We speculate that PCA involvement is an independent factor in posterior hemorrhage for the following reasons. First, stenosis of the PCA might induce ischemia in the posterior half of the brain. Accordingly, this ischemia might increase the hemodynamic burden on choroidal collaterals, which is not always revealed through angiography, resulting in posterior hemorrhage. Second, PCA involvement might also promote the development of thalamic anastomosis, increasing the probability of posterior hemorrhage. The latter phenomenon has been designated by the senior author as "posterior moyamoya."

The present results might provide a clue to the mechanism of high rebleeding risk related to posterior hemorrhage. ${ }^{14}$ Choroidal anastomosis, which is the most posteriorly distributed of the various types, might, by its nature, present the highest risk of rerupture among the fragile collaterals. Whether choroidal anastomosis is a true predictor of rebleeding should be tested in further longitudinal analyses.

Focusing on choroidal anastomosis as a risk factor for hemorrhage could be of greater clinical importance because the factor might be modifiable with treatment. Jiang et al. reported that the choroidal collateral vessel can shrink after bypass surgery. ${ }^{7}$ The mechanism of such shrinkage might be reduction of hemodynamic burden in the choroidal collateral vessel, resulting from augmentation of cerebral blood flow in the cortex. The shrinkage might thus depend on the location onto which the bypass had been grafted. If this hypothesis were to prove true, preoperative assessment focusing on choroidal collaterals would facilitate selection of a recipient site that reduces the hemodynamic burden on the collaterals, although recipient site selection depends primarily on the length of the donor scalp artery and the size of the cortical arteries.

Although the present study used the robust data set of a strictly designed prospective randomized controlled trial, it has 3 specific limitations. First, the causal association between choroidal anastomosis or PCA involvement and posterior hemorrhage is tentative because of the cross-sectional design of the study. Second, the reliability of the angiographic classification of anastomotic collaterals, which remains to be assessed, could affect the results. Third, despite the care taken to eliminate biases, a post hoc analysis cannot be free from them. Further study is needed to overcome these limitations.

\section{Conclusions}

Choroidal anastomosis might be responsible for posterior hemorrhage, a predictor of rebleeding in hemorrhagic moyamoya disease. PCA involvement is another significant factor associated with posterior hemorrhage. The concept of choroidal anastomosis might help clarify the mechanism of posterior hemorrhage and serve as a modifiable risk factor for rebleeding, subject to verification in further analyses.

\section{Acknowledgments}

The JAM Trial has been funded since 1999 by a grant from the Japanese Ministry of Health, Labour and Welfare as a major project of the Research Committee on Spontaneous Occlusion of the Circle of Willis (moyamoya disease).

\section{References}

1. Baltsavias G, Khan N, Filipce V, Valavanis A: Selective and superselective angiography of pediatric moyamoya disease angioarchitecture in the posterior circulation. Interv Neuroradiol 20:403-412, 2014

2. Fukui M: Guidelines for the diagnosis and treatment of spontaneous occlusion of the circle of Willis ('moyamoya' disease). Research Committee on Spontaneous Occlusion of the Circle of Willis (Moyamoya Disease) of the Ministry of Health and Welfare, Japan. Clin Neurol Neurosurg 99 (Suppl 2):S238-S240, 1997

3. Funaki T, Fushimi Y, Takahashi JC, Takagi Y, Araki Y, Yoshida K, et al: Visualization of periventricular collaterals in moyamoya disease with flow-sensitive black-blood magnetic resonance angiography: preliminary experience. Neurol Med Chir (Tokyo) 55:204-209, 2015

4. Funaki T, Takahashi JC, Takagi Y, Yoshida K, Araki Y, Kikuchi T, et al: Impact of posterior cerebral artery involvement on long-term clinical and social outcome of pediatric moyamoya disease. J Neurosurg Pediatr 12:626-632, 2013

5. Funaki T, Takahashi JC, Yoshida K, Takagi Y, Fushimi Y, Kikuchi T, et al: Periventricular anastomosis in moyamoya disease: detecting fragile collateral vessels with MR angiography. J Neurosurg 124:1766-1772, 2016

6. Irikura K, Miyasaka Y, Kurata A, Tanaka R, Fujii K, Yada K, et al: A source of haemorrhage in adult patients with moyamoya disease: the significance of tributaries from the choroidal artery. Acta Neurochir (Wien) 138:1282-1286, 1996 
7. Jiang H, Ni W, Xu B, Lei Y, Tian Y, Xu F, et al: Outcome in adult patients with hemorrhagic moyamoya disease after combined extracranial-intracranial bypass. J Neurosurg 121:1048-1055, 2014

8. Marinković S, Gibo H, Filipović B, Dulejić V, Piscević I: Microanatomy of the subependymal arteries of the lateral ventricle. Surg Neurol 63:451-458, 2005

9. Miyamoto S, Kikuchi H, Karasawa J, Nagata I, Ikota T, Takeuchi S: Study of the posterior circulation in moyamoya disease. Clinical and neuroradiological evaluation. J Neurosurg 61:1032-1037, 1984

10. Miyamoto S, Yoshimoto T, Hashimoto N, Okada Y, Tsuji I, Tominaga T, et al: Effects of extracranial-intracranial bypass for patients with hemorrhagic moyamoya disease: results of the Japan Adult Moyamoya Trial. Stroke 45:1415-1421, 2014

11. Morioka M, Hamada J, Kawano T, Todaka T, Yano S, Kai Y, et al: Angiographic dilatation and branch extension of the anterior choroidal and posterior communicating arteries are predictors of hemorrhage in adult moyamoya patients. Stroke 34:90-95, 2003

12. Saito R, Kumabe T, Sonoda Y, Kanamori M, Mugikura S, Takahashi S, et al: Infarction of the lateral posterior choroidal artery territory after manipulation of the choroid plexus at the atrium: causal association with subependymal artery injury. J Neurosurg 119:158-163, 2013

13. Suzuki J, Kodama N: Moyamoya disease-a review. Stroke 14:104-109, 1983

14. Takahashi JC, Funaki T, Houkin K, Inoue T, Ogasawara K, Nakagawara J, et al: Significance of the hemorrhagic site for recurrent bleeding: prespecified analysis in the Japan Adult Moyamoya Trial. Stroke 47:37-43, 2016
15. Takahashi S, Fukasawa H, Ishii K, Sakamoto K: The anterior choroidal artery syndrome. I. Microangiography of the anterior choroidal artery. Neuroradiology 36:337-339, 1994

16. Takahashi S, Suzuki M, Matsumoto K, Ishii K, Higano S, Fukasawa H, et al: Extent and location of cerebral infarcts on multiplanar MR images: correlation with distribution of perforating arteries on cerebral angiograms and on cadaveric microangiograms. AJR Am J Roentgenol 163:1215-1222, 1994

\section{Disclosures}

The authors report no conflict of interest concerning the materials or methods used in this study or the findings specified in this paper.

\section{Author Contributions}

Conception and design: Miyamoto, Funaki. Acquisition of data: Funaki, Takahashi, Kuroda, Takeuchi, Fujimurac, Miyamoto. Analysis and interpretation of data: all authors. Drafting the article: Funaki. Critically revising the article: all authors. Reviewed submitted version of manuscript: all authors. Approved the final version of the manuscript on behalf of all authors: Miyamoto. Statistical analysis: Tomata.

\section{Correspondence}

Susumu Miyamoto, Department of Neurosurgery, Graduate School of Medicine, Kyoto University, 54 Shogoin Kawahara-cho, Sakyo-ku, Kyoto 606-8507, Japan. email: miy@kuhp.kyoto-u. ac.jp. 